OPEN ACCESS

Edited by:

Eric Altermann,

AgResearch Ltd., New Zealand

Reviewed by:

Uri Gophna,

Tel Aviv University, Israe

A. Murat Eren,

University of Chicago, USA

${ }^{*}$ Correspondence:

Nicholas Chia

chia.nicholas@mayo.edu

Specialty section:

This article was submitted to Evolutionary and Genomic

Microbiology,

a section of the journa

Frontiers in Microbiology

Received: 01 April 2016 Accepted: 09 May 2016

Published: 26 May 2016

Citation:

Jeraldo P, HernandezA, Nielsen HB, Chen X, White BA, Goldenfeld N,

Nelson H, Alhquist $D$, Boardman L and Chia N (2016) Capturing One of the Human Gut Microbiome's Most Wanted: Reconstructing the Genome of a Novel Butyrate-Producing, Clostridial Scavenger from Metagenomic Sequence Data.

Front. Microbiol. 7:783 doi: 10.3389/fmicb.2016.00783

\section{Capturing One of the Human Gut Microbiome's Most Wanted: Reconstructing the Genome of a Novel Butyrate-Producing, Clostridial Scavenger from Metagenomic Sequence Data}

Patricio Jeraldo 1,2,3, Alvaro Hernandez ${ }^{4}$, Henrik B. Nielsen ${ }^{5}$, Xianfeng Chen 6 , Bryan A. White ${ }^{3,7}$, Nigel Goldenfeld ${ }^{3,8}$, Heidi Nelson ${ }^{1,2}$, David Alhquist $^{9}$, Lisa Boardman ${ }^{9}$ and Nicholas Chia ${ }^{1,2,10 *}$

${ }^{1}$ Microbiome Program, Center for Individualized Medicine, Mayo Clinic, Rochester, MN, USA, ${ }^{2}$ Department of Surgery, Mayo Clinic, Rochester, MN, USA, ${ }^{3}$ Carl R. Woese Institute for Genomic Biology, University of Illinois at Urbana-Champaign, Urbana, IL, USA, ${ }^{4}$ Roy J. Carver Biotechnology Center, University of Illinois at Urbana-Champaign, Urbana, IL, USA, ${ }^{5}$ Center for Biological Sequence Analysis, Department of Systems Biology, Technical University of Denmark, Kongens Lyngby, Denmark, ${ }^{6}$ Department of Health Sciences Research, Mayo Clinic, Rochester, MN, USA, ' Department of Animal Sciences, University of Illinois at Urbana-Champaign, Urbana, IL, USA, ${ }^{8}$ Department of Physics, University of Illinois at Urbana-Champaign, Urbana, IL, USA, ${ }^{\circ}$ Division of Gastroenterology and Hepatology, Mayo Clinic, Rochester, MN, USA,

${ }^{10}$ Department of Physiology and Biomedical Engineering, Mayo Clinic, Rochester, MN, USA

The role of the microbiome in health and disease is attracting great attention, yet we still know little about some of the most prevalent microorganisms inside our bodies. Several years ago, Human Microbiome Project (HMP) researchers generated a list of "most wanted" taxa: bacteria both prevalent among healthy volunteers and distantly related to any sequenced organisms. Unfortunately, the challenge of assembling highquality genomes from a tangle of metagenomic reads has slowed progress in learning about these uncultured bacteria. Here, we describe how recent advances in sequencing and analysis allowed us to assemble "most wanted" genomes from metagenomic data collected from four stool samples. Using a combination of both de novo and guided assembly methods, we assembled and binned over 100 genomes from an initial data set of over 1,300 Gbp. One of these genome bins, which met HMP's criteria for a "most wanted" taxa, contained three essentially complete genomes belonging to a previously uncultivated species. This species is most closely related to Eubacterium desmolans and the clostridial cluster IV/Clostridium leptum subgroup species Butyricicoccus pullicaecorum (71-76\% average nucleotide identity). Gene function analysis indicates that the species is an obligate anaerobe, forms spores, and produces the antiinflammatory short-chain fatty acids acetate and butyrate. It also appears to take up metabolically costly molecules such as cobalamin, methionine, and branch-chained amino acids from the environment, and to lack virulence genes. Thus, the evidence is consistent with a secondary degrader that occupies a host-dependent, nutrient- 
scavenging niche within the gut; its ability to produce butyrate, which is thought to play an anti-inflammatory role, makes it intriguing for the study of diseases such as colon cancer and inflammatory bowel disease. In conclusion, we have assembled essentially complete genomes from stool metagenomic data, yielding valuable information about uncultured organisms' metabolic and ecologic niches, factors that may be required to successfully culture these bacteria, and their role in maintaining health and causing disease.

Keywords: microbiome, metagenomics, Butyricicoccus, binning, genome assembly

\section{INTRODUCTION}

The vast majorities of bacteria within and around us have not been successfully cultured (Torsvik et al., 1990; Fierer et al., 2008), and are thus difficult to identify, much less study. The field of metagenomics seeks to address this problem by directly accessing the genomes of uncultured organisms and analyzing their contents (Handelsman et al., 1998; Handelsman, 2004). On the one hand, recent sequencing and analytical advances and efforts such as the Human Microbiome Project (HMP) (Gevers et al., 2012) and the Metagenomics of the Human Intestinal Tract (MetaHIT) project (Qin et al., 2010) have allowed us to learn more about our "microbial dark matter" (Lok, 2015). On the other hand, some of the most common microbial residents of our bodies remain elusive, leading Fodor et al. (2012) to compose a "most wanted" list of HMP taxa. Four years ago, they issued a challenge to the research community to sequence the genomes of these "most wanted" organisms, which were prioritized based on their frequency in healthy HMP volunteers and their distance to already sequenced organisms. Simply by characterizing these organisms' gene repertoires, we can glean key information about their functional niches in the body: what compounds they synthesize, how they use the metabolites present in their environment, the mechanisms they use to interact with or invade our cells, and whether they are resistant to antibiotics.

Although there is consensus that analyzing the genomes of these taxa is essential to understanding the role of the microbiome in maintaining health, the challenges associated with assembling specific genomes from the complex morass of metagenomic data have slowed progress toward this goal. Recently, MetaHIT researchers made headway by identifying sets of coabundant gene groups (CAGs) in metagenomic data from multiple samples, and assigning these CAGs to individual microbial species (Nielsen et al., 2014). The next logical goal is to use a similar approach to assemble whole genomes from metagenomic data. Although initially it was only possible for less complex microbial communities, assembling genomes directly from the environment has gained traction thanks to the improved approaches and software platforms, such as differential coverage and k-mer-based binning methods, which open potential avenues for their increased use in the study of ecosystems such as the gut microbiome (Albertsen et al., 2013; Alneberg et al., 2013; Sharon and Banfield, 2013; Eren et al., 2015).

We harnessed these recent advances in sequencing and metagenomic analysis to analyze the microbes present in four stool samples and to obtain three essentially complete genomes belonging to an uncharacterized species that met the HMP's "most wanted" criteria. Based on the metabolic profiles obtained from these genomes, we predict that this species is a scavenger dependent on metabolites produced by other gut bacteria, that successful culture may depend on supplementation with methionine and cobalamin, and also that the bacterium produces the anti-inflammatory small-chain fatty acids (SCFAs) acetate and butyrate, making it a potential candidate for probiotic therapy. Thus, we show that, using readily accessible methods, it is possible to assemble the genomes of novel species from metagenomic data and to gain insight into their ecological niches, nutrient requirements, and potential roles in maintaining health.

\section{MATERIALS AND METHODS}

\section{Subject Consent and Sampling}

Four stool samples were collected prior to colonoscopy as part of a broader ongoing study of patients at the Mayo Clinic. Two (A254 and K4410) were from subjects diagnosed with colon cancer, and two (N15 and N54) were from subjects with a negative colonoscopy. This study was reviewed and approved by the Institutional Review Board at Mayo Clinic under protocol numbers 10-004833 and 10-006009, and written informed consent was obtained from all participants.

\section{DNA Extraction}

Genomic DNA (gDNA) was extracted from the stool samples using the PowerSoil DNA isolation Kit (MO BIO Laboratories, Carlsbad, CA, USA). Briefly, for each sample, we created 24 aliquots, each containing approximately $150-200 \mathrm{mg}$ stool. These aliquots were then processed together in a single batch. After adding $60 \mu \mathrm{l}$ buffer $\mathrm{C} 1$ to each aliquot, the samples were homogenized in PowerBead tubes on the FastPrep-24 (MP Biomedicals, Santa Ana, CA, USA; settings: $S=4, T=40 \mathrm{~s}$ ). Next, the supernatant was processed following the manufacturer's protocol with a few modifications: $70 \mu \mathrm{l}$ buffer $\mathrm{C} 6$ was loaded to the spin filter, and the product was loaded for a second elution to increase its concentration. All eluted products from the same stool sample were then pooled and quantified with the Qubit fluorometer (Invitrogen, Carlsbad, CA, USA). In the case of insufficient yield, more stool aliquots were processed, to yield at least $1 \mu \mathrm{g}$ gDNA from each sample. 


\section{Construction of Shotgun and Mate-Pair Libraries}

To minimize misassemblies due to repeat regions, we created both shotgun and mate-pair libraries, which allowed us to sequence genomic regions separated by short and long distances. The multiple library fragment size ranges allowed us to reach past repeat regions, and multiple mate-pair size selections granted us access to extra sources of validation for scaffolding. The libraries were constructed at the Roy J. Carver Biotechnology Center, University of Illinois at Urbana-Champaign (UIUC), which also carried out sequencing. Shotgun gDNA libraries were constructed with the Library Preparation Kit from Kapa Biosystems (Wilmington, MA, USA) from $1 \mu \mathrm{g}$ of DNA sonicated with a Covaris M220 (Woburn, MA, USA). These libraries were loaded onto a $2 \%$ agarose gel, and fragments 400-600 bp in length were selected. Final libraries were run on high-sensitivity DNA chips (Agilent, Santa Clara, CA, USA) to determine the average fragment size and to confirm the presence of DNA in the expected size range. The libraries were also quantitated by qPCR on a BioRad CFX Connect Real-Time System (Hercules, CA, USA).

To generate reads with longer insert sizes, mate-pair libraries were prepared with the Nextera Mate-Pair Sample Preparation Kit (Illumina; San Diego, CA, USA). Briefly, $10 \mu \mathrm{g}$ high-quality gDNA was subjected to two tagmentation reactions and run on a $0.6 \% \mathrm{Mb}$ agarose gel. Genomic fragments $4-6 \mathrm{~kb}, 8-$ $12 \mathrm{~kb}$, and $15-20 \mathrm{~kb}$ in size were selected, purified on an EluTrap (GE Healthcare Life Sciences; Piscataway, NJ, USA), and circularized. The circles were sonicated with a Covaris M220 (Covaris, Woburn, MA, USA) and enriched for fragments containing the biotinylated circularization adapter. Enriched fragments were then end-repaired, A-tailed, adaptered, and PCR amplified with the TruSeq DNA Sample Prep kit (Illumina). Final libraries checked for size and quantitated as described above.

\section{Sequencing on an Illumina HiSeq2500}

The libraries were sequenced on an Illumina HiSeq2500. DNA fragments were sequenced for 151 cycles from each end using TruSeq Rapid SBS v1 sequencing kits. Then, the raw.bcl files were converted into demultiplexed compressed fastq files using bcl2fastq v1.8.2 Conversion Software (Illumina).

\section{Preprocessing of Sequenced Reads}

\section{Removal of Human Host Reads}

First, we removed raw reads identified as human sequences. To do so, we ran Kraken v0.10.4-beta (Wood and Salzberg, 2014) trained with the Homo sapiens reference from the 1000 Genomes Project, bacterial and archaeal genomes from NCBI's "Bacteria" collection, and viral genomes from NCBI's "Viruses" collection. This step was repeated as necessary during read processing, as described below.

\section{Quality Trimming and Adapter Removal}

Next, we removed sequencing adapters and trimmed low-quality bases from the reads using Trimmomatic v0.32 (Bolger et al., 2014) and the following settings: ILLUMINACLIP:adapters.fasta:2:30:10 LEADING:3 TRAI LING:3 MAXINFO:100:0.1 MINLEN:60. The "adapters.fasta" file, which contains a description of the Illumina TruSeq 3 and Nextera adapters, is distributed with the Trimmomatic package. Singleton, orphan reads (i.e., reads that lost their mate during the filtering step) were retained as single reads.

\section{Error Correction}

Error correcting reads increases assembly quality by removing $\mathrm{k}$-mers when there is statistical or quality evidence they come from sequencing errors, and this step is usually included in singlegenome assemblers such as SPAdes, but not to our knowledge for metagenomic assemblers. We performed basic error correction of the reads using the error correction step of the SGA assembler (Simpson and Durbin, 2012). For preprocessing, the following command was used: sga preprocess -dust -no-primer-check $\mathrm{m} \mathrm{60}$; for correction, we used the command sga correct $-\mathrm{k}$ 55 -discard -learn.

\section{Digital Read Coverage Normalization}

Read coverage normalization is a step needed when the assembly process would require computer memory resources that exceed the available capacity, at the expense of discarding some of the read data, and deep metagenomes like the one in this study can easily exceed this capacity. Read coverage was independently digitally normalized with khmer v1.3 (Crusoe et al., 2015). For each of a sample's libraries, we performed a single-pass digital normalization to $60 \mathrm{X}$ using the command normalize-bymedian.py $-\mathrm{k} 20-\mathrm{N} 4-\mathrm{x} 3 \mathrm{e} 10-\mathrm{C} 60$, with a false-positive rate less then 0.001 (we increased the value of $-x$ if the false-positive rate was larger than 0.001). Then we performed abundance filtering using the command filter-abund.py $-\mathrm{V}$.

\section{Metagenomic Assembly}

\section{Removal of Host Reads and Assembly}

We performed a second pass of Kraken on the normalized reads to remove human host reads.

We then assembled each sample separately, using all available libraries, with Ray-Meta version 2.3.1 (Boisvert et al., 2012), using a k-mer value of 39 . The annotation step of the program was skipped. Finally, we ran Kraken a third time to discard newly identified human host reads. By using the normalized read libraries, we ensured RAM usage during assembly was within the capacity of our systems.

\section{De Novo Binning and Reassembly}

To perform de novo binning, we followed the steps outlined by Albertsen et al. (2013). Briefly, we annotated the assembled contigs using per-sample coverage information, consensus taxonomy, GC content, and tetranucleotide spectrum information (for information on how we calculated GC content and tetranucleotide spectrum information, see below). We then used the differential coverage information, taxonomy, and GC content to manually bin clusters of contigs putatively belonging to the same organism; tetranucleotide spectrum values 
were used to remove contigs dissimilar to the bulk of other contigs in a bin.

\section{Coverage annotation of assembled contigs}

We extracted contigs larger than or equal to $1,000 \mathrm{bp}$ and mapped error-corrected, un-normalized reads from all samples onto the contigs using BWA-mem version 0.7.6a (Li and Durbin, 2009). We then calculated per-sample coverage using SAMtools version $0.1 .19-44428 \mathrm{~cd}$ ( $\mathrm{Li}$ et al., 2009) and bedtools version 2.16.2 (Quinlan, 2014).

\section{Taxonomy annotation of assembled contigs}

For contigs longer than $1,000 \mathrm{bp}$, we predicted genes using MetaProdigal version 2.60 (Hyatt et al., 2012). We queried the peptide sequences of the predicted genes against the RefSeq protein database (performed on October 29, 2013) using USEARCH version 6.0.307 (Edgar, 2010) in uBlast mode, with an e-value of 1e-5, maxhits set to 5 , and accel at 0.9 (command: usearch6.0.307_i86linux64 -ublast queries.faa -db refseq.udb evalue 1e-5 -maxhits 5 -accel 0.9 -blast6out output.txt). We then parsed the USEARCH output to determine the taxonomy lineage of the lowest common ancestor (LCA) for each predicted gene using blast2lca (Altschul et al., 1990; Pignatelli, 2014) against the NCBI taxonomy (obtained on October 29, 2013) and summarized the LCA for a contig using the voting scheme implemented in the script hmm.majority.vote.pl, used in Albertsen et al. (2013).

\section{GC and tetranucleotide spectrum annotation}

We calculated per-contig GC content and the canonical (strandindependent) tetranucleotide (4-mer) spectrum using custom scripts. Instead of saving the raw 4-mer counts per contig or the frequency of each 4-mer per contig, we saved the $z$-score of each 4-mer per contig.

\section{Manual identification of bins}

As outlined in Albertsen et al. (2013), we created differential coverage plots annotated with the taxonomy and sequence data described above. To reduce clutter, we split the data by phylum (Firmicutes, Bacteroidetes, Actinobacteria, Proteobacteria, and "other phyla") and then examined the differential coverage plots. In the case of the Firmicutes and Bacteroidetes, we split the data even further, to the order or family level when possible. Once we identified a set of contigs, we used the corresponding tetranucleotide spectra to perform principal components analysis (PCA), plotting the contigs against the first and second dimensions. We selected the contigs that clustered together, with the presumption that outliers did not belong in the bins.

\section{Mapping and reassembly}

We mapped error-corrected, un-normalized reads from all eight libraries (one shotgun and one mate-pair library for each sample) onto the preliminary bins using BWA-mem. We have found that combining velvet and SPAdes produces more complete bin assemblies. Thus, we performed a first-pass assembly using velvet version 1.2.10 (Zerbino and Birney, 2008) with $\mathrm{k}$-mer values from $k=19$ to $k=99$, retaining contigs longer than $1,000 \mathrm{bp}$. Then, we performed a second-pass assembly using SPAdes version 3.1.0 (Bankevich et al., 2012), passing the velvet contigs from the best assembly (containing the longest contig) to SPAdes using the "untrusted-contig" parameter. Using Prodigal and HMMER version 3.0 (Eddy, 2011), we identified each bin's essential genes using the hidden Markov models (HMM) used for essentiality tests in Albertsen et al. (2013), and we declared a bin "essentially complete" if we found 100 or more unique genes from this HMM set, which contains profiles for 111 genes. We also searched for $16 \mathrm{~S}$ rRNA reads using RNAmmer (Lagesen et al., 2007). Finally, if $16 \mathrm{~S}$ rRNA and $r p o B$ sequences were present among the essential genes, we used them to taxonomically identify the bin and also as potential markers for contaminant contigs. At this point, the bins were ready for further contamination and completeness checks, outlined below.

\section{Supervised Binning and Reassembly Genome references}

First, we selected references for guided reconstruction of genomes: 741 metagenomic species (MGSs) identified as part of a large collection of metagenomic libraries of the human intestinal tract (Nielsen et al., 2014). The MGSs are groups of at least 700 genes whose abundance across samples is highly correlated; they served as seeds for the progressive assembly of genomes.

\section{Read mapping and rRNA removal}

Next, we ensured that the highly conserved rRNA operon, which receives high coverage, did not interfere with the assembly process. The reason is that rRNA is highly conserved across species, and when mapping reads onto the MGS bins there were regions of extremely high coverage which may result in chimeric contigs. To avoid this, we decided to discard these reads for this assembly step. To do so, we mapped the un-normalized reads described earlier onto the genes of each MGS bin using BWA, discarding unmapped read pairs. We then mapped the resulting reads onto the SILVA database version 119 (Quast et al., 2013), which contains $16 \mathrm{~S}$ and $23 \mathrm{~S}$ rRNA reads, and removed reads that aligned to the SILVA reference.

\section{Bin assembly and cleanup}

We assembled the bin reads using SPAdes with k-mer values of " $21,33,55,77$," " $21,33,55,77,99$," and " $21,33,55,77,99,127$," retaining the assembly with the longest assembled contig for further processing. We then removed contigs shorter than $1,000 \mathrm{bp}$ and with reported coverage less than $2 \mathrm{X}$. Putative contaminant sequences (i.e., those not belonging to the bins) were removed using BioBloom tools version 2.0.6 (Chu et al., 2014), trained with either the original MGS gene bin or with the corresponding published assembly, if available. We continued removing putative contaminants using CheckM version 0.9.7 (Parks et al., 2015) by calculating the bin distribution of GC content, coding density, and tetranucleotide spectrum, and then removing outliers - -contigs falling outside of the $95 \%$ Confidence Intervals for these distributions- - through the "checkm outliers" command. After this step, we discarded any bin with less than 100,000 bp assembled.

\section{Reiteration of the process}

In order to continue improving the assemblies and removing low quality contigs, the resulting bins were used to reiterate the steps 
above, starting with mapping. Briefly, we mapped un-normalized reads onto the bin obtained from the previous iteration, mapped against SILVA 119 to discard rRNA, reassembled with SPAdes, filtered out short and low-coverage contigs, removed putative contaminants using BioBloom tools trained with the bin obtained from the previous iteration, and removed outliers using CheckM. After repeating this process three times, we found little additional changes on both contigs added and low quality assemblies removed and therefore we terminate the iterative clean-up process.

\section{Extension of assembled contigs}

To complete the assembly process, we use a specialized assembler that extends the contigs from their tips using the unassembled reads. For some of the contigs, this has the effect of re-introducing the rRNA operons, and by requiring high mapping identities to the ends of the contigs, the process will likely not introduce misassemblies. To extend the contigs obtained from the binning process, we used the PRICE genome assembler version 1.2 (Ruby et al., 2013), which maps reads onto the ends of contigs and iteratively extends them. We used the normalized paired-end reads and ran the program for 10 cycles, with parameters -fpp reads_R1.fastq reads_R2.fastq 50095 -icf my_bin.fasta $11 \quad 1 \quad 5$-nc 10 -dbmax 72 -mol 30 -tol 20 -mpi 90 -target 900 , extending input contigs but not creating new ones. We discarded bins in which the extension process was not finished. In addition, when PRICE extends contigs with highly repetitive regions, it appears the memory usage increases until all available memory is saturated. Consequently, the bins containing a high number of repeat regions may not be present at all. For this reason, we also discarded bins that used excessive memory.

\section{Completeness check and final contamination removal}

Using CheckM, we estimated each bin's completeness and contamination level, and we retained bins with completeness greater than $70 \%$ and contamination less than $5 \%$ for further processing. To assign taxons, we ran Prodigal to predict genes, used USEARCH to find matches in RefSeq in uBlast mode, and used blast2lca to estimate an LCA taxonomic assignment for the bins. We then ran ProDeGe version 2.2 (Tennessen et al., 2015) on each bin to remove additional putative contaminants, passing the estimated taxonomy assignment as a parameter.

\section{Identification of the "Most Wanted" Taxa in Stool Samples}

Following the same criteria as set forth by Fodor et al. (2012), we began by determining which organisms were present in the Midwest Reference Panel (MWRP) (Chen et al., 2016), a recently described gut microbiome sample representative of the volunteers who gave stool samples for this project. Operational taxonomic units (OTUs) present in the MWRP were searched against $16 \mathrm{~S}$ genes extracted from 12,724 human-associated genomes from the Genomes Online Database Human (GOLDHuman) version 5 (as of September 28, 2015) (Reddy et al., 2015) and also from HMP strains (Fodor et al., 2012), and sequence identity was calculated using PyNAST (Caporaso et al., 2010). In addition, the prevalence of OTUs was calculated by counting the number of MWRP samples in which each OTU was present. This allowed us to separate high-priority, medium priority, and low-priority genomes based on genetic novelty and prevalence in the human gut. As a simple example, a genome that has unique characteristics and is present in all of us would have the highest priority. As per the Fodor et al. (2012) criteria, "most-wanted" OTUs were those with $90 \%$ or less similarity to a sequenced genome and a prevalence greater than or equal to $20 \%$ in the MWRP cohort. Medium priority genomes were those between 90 and $97 \%$ similarity to a sequenced genome and prevalence greater than or equal to $20 \%$ in the MWRP cohort. The remaining genomes were low priority.

Next, we determined which high-priority MWRP OTUs were present in the metagenomic data from the four stool samples we collected. To do this, $16 \mathrm{~S}$ reads extracted from the assembly were clustered against the MWRP OTUs, and reads were deemed a hit if there was $97 \%$ or higher sequence similarity. Genomic bins with no identity to an OTU in the MWRP were defined as zero prevalence. We also used completeness as a criterion to decide which bin to characterize further, with essentially complete bins (as defined above) receiving higher priority. Based on "mostwanted" and completion status, we chose three genomes from bin MGS46 for additional study. They were essentially complete, $90.0 \%$ similar to the closest publicly available genome, and present in $97.5 \%$ of MWRP volunteers (Figure 1, Supplementary Table S1).

\section{Annotation of Genomes}

Genomes were annotated using RASTtk (Brettin et al., 2015) through PATRIC (Snyder et al., 2007). Functional characterization was also performed using PATRIC.

\section{Phylogeny and Functional Comparison}

A 16S rRNA phylogeny and two types of whole-genome phylogeny were constructed to determine where the three selected genomes fell on the tree of life. These different types of phylogeny provide complementary information. 16S rRNA phylogenies allow deep, long-range reconstructions of evolutionary relationships but lack resolution below the species level. By contrast, whole-genome clustering using BLASTbased average nucleotide identity (ANIb) provides strain-level resolution and correlates well with DNA-DNA hybridization (DDH) cutoffs for species $(70 \% \mathrm{DDH}$ is equivalent to $95 \%$ ANIb). ANIb phylogenies become unreliable for identities lower than $80 \%$, however, due to low levels of the overlap necessary to calculate identity (Rosselló-Móra and Amann, 2015). An alternate method bridges the gap between ANIb and 16S rRNA phylogenies by using the whole genome to construct a tetranucleotide spectrum, which is used to calculate distances with reliability that extends beyond the species level (Teeling et al., 2004).

The $16 \mathrm{~S}$ phylogeny was constructed by aligning the $16 \mathrm{~S}$ sequences of the reference and assembled organisms using Infernal, with the bacterial covariance model from RDP (Cole et al., 2009). An approximate maximum likelihood phylogeny was computed using FastTree (Price et al., 2010) with the parameters -nt -gtr -gamma -slownni -spr 4 -mlacc 2. The ANIb phylogeny 
A
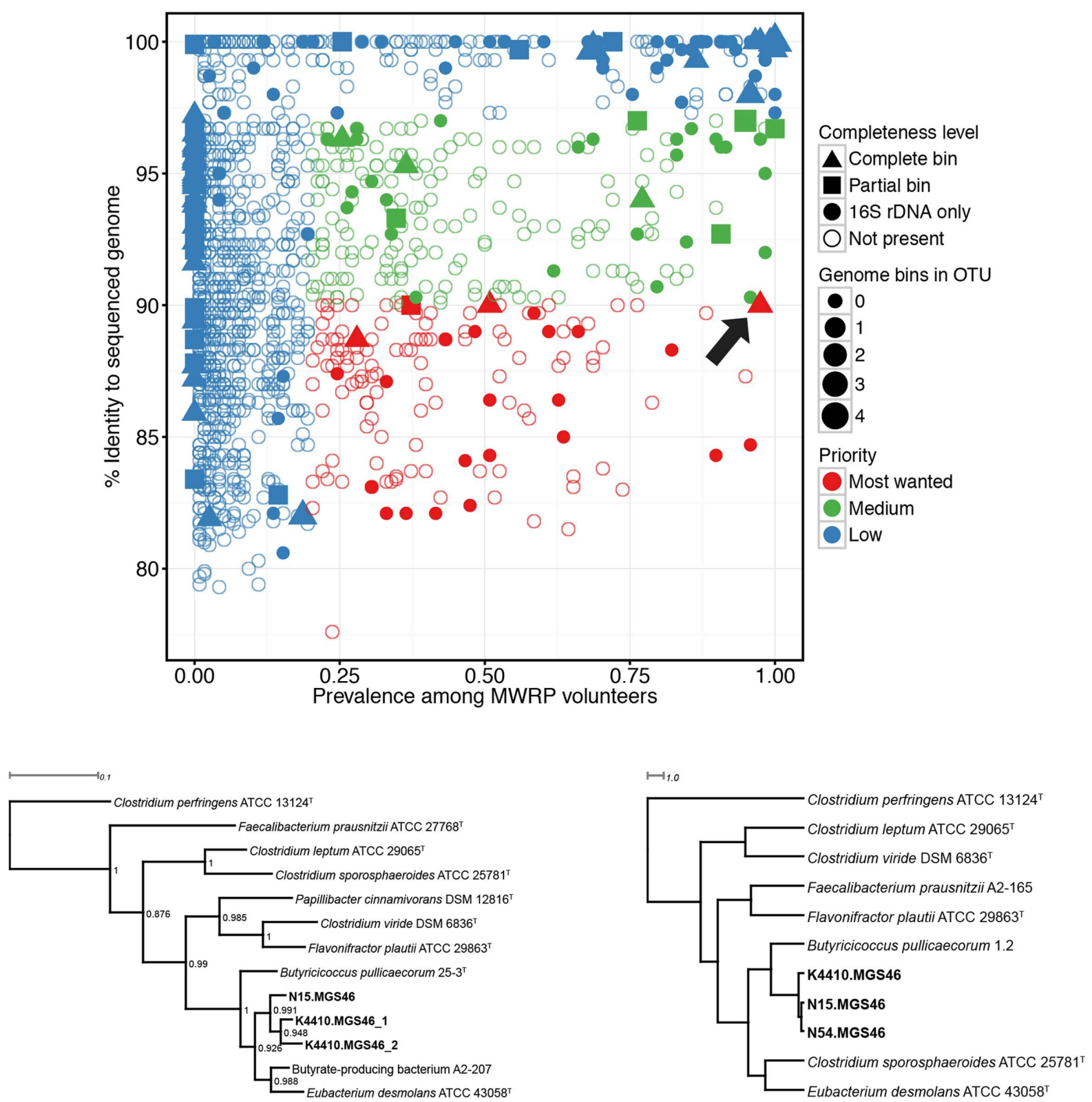

FIGURE 1 | (A) Numerous "most wanted" taxa were present in samples from the Midwestern Reference Panel (MWRP), and one essentially complete "most wanted" bin was present in metagenomic data gathered from four stool samples. $16 \mathrm{~S}$ amplicons were used to establish the prevalence of various taxa among the MWRP volunteers and the distance of those taxa from already sequenced organisms, as determined by matches to the Genomes Online Database Human-associated genomes (GOLD-Human) or the Human Microbiome Project Strains. The size of each bin indicates the number of hits to that OTU in the MWRP. As in Fodor et al. (2012), prevalent taxa (>20\%) that were not closely related to already sequenced organisms $(<90 \%)$ were assigned highest priority. Colors represent "most wanted" status: red, high priority; green, medium priority; blue, low priority. Filled shapes indicate the binned genomes in our stool metagenomic data set that matched operational taxonomic units (OTUs) from the MWRP with 97\% or higher similarity. Shapes indicate the completion status of the binned genome in our metagenomic data set: triangle, complete sequence data; square, partial sequence data; circle, $16 S$-only sequence data. The arrow points to the Butyricicoccus sp. B MC bin, which we selected for further study. (B) Phylogenies show that the closest relatives of the three assembled genomes (Butyricicoccus sp. B MC N15, -N54, and -K4410) within the essentially complete "most wanted" bin are clostridial cluster IV/Clostridium leptum subgroup strains. Left: 16S rRNA phylogeny of the assembled genomes together with type strains from species of the clostridial IV/Clostridium leptum subgroup. Clostridium perfringens is an outgroup, and bootstrap values are shown for each node. Note: N54 is missing because its $16 \mathrm{~S}$ sequence is not available. Right: Tetranucleotide spectrum-based whole-genome phylogeny of the three reconstructed genomes together with genomes from the clostridial cluster IV/C. leptum subgroup type species. Both phylogenies identify the nearest neighbors as Butyricicoccus pullicaecorum and Eubacterium desmolans. 
was computed by using JSpecies (Richter and Rosselló-Móra, 2009) and then performing hierarchical clustering using the apcluster package. The tetranucleotide whole-genome phylogeny was constructed by computing the canonical tetranucleotide spectra of all genomes (Teeling et al., 2004), transforming these data into $z$-scores and calculating the Euclidian distance, and then performing exemplar-based agglomerative clustering (a hierarchical clustering method) using the $\mathrm{R}$ package apcluster (Frey and Dueck, 2007; Bodenhofer et al., 2011).

A pangenome analysis of the three assembled genomes and their two nearest neighbors was performed using the GView Server (Petkau et al., 2010).

Usage of the clusters of orthologous groups (COGs/NOGs) (Huerta-Cepas et al., 2015) was computed by mapping gene annotation onto COG categories and then normalizing by the total number of hits per genome.

A functional hierarchy was calculated by determining the FIGfams functional families (Meyer et al., 2009) present in each genome and then calculating the Jaccard dissimilarity between genomes based on presence/absence information for each family. Hierarchical clustering was performed using the apcluster package.

Finally, pathway analysis was performed using the Protein Family Sorter tool from the PATRIC service. PATRIC was also used to scan for virulence genes.

\section{Data Deposition}

Raw sequence data and assembled genomes were deposited in NCBI with human reads removed under BioProject accession number PRJNA312222.

\section{RESULTS}

We assembled a total of 90 genomic bins from the sequence data obtained from the four stool samples, and from these bins we identified 59 essentially complete genomes (see Materials and Methods for a description of how completeness was calculated). These genomes, including 24 previously announced by Jeraldo et al. (2015) but not yet described, came from diverse genera. Some genomes represented genera known to dominate the gut, including Bacteroides $(n=2)$, Clostridium $(n=3)$, Eubacterium $(n=2)$, and Ruminococcus $(n=3)$. Lactobacillus $(n=2)$, a genus known for its health promoting effects, was also represented. Other, less-common genera included Acinetobacter $(n=1)$; Akkermansia $(n=1)$, some species that are associated with healthier metabolic status (Dao et al., 2016); bile-tolerant Alistipes $(n=1)$; Burkholderia $(n=1)$; Carnobacterium $(n=2)$; Odoribacter $(n=3)$, increased levels of which, along with Bacteroides, have been linked to colon tumorigenesis in mice (Zackular et al., 2013); and Roseburia $(n=1)$, a genus enriched by dietary whole grains (Martínez et al., 2013). In addition, we identified a genome belonging to the Melainabacteria $(n=1)$, a proposed class in the Cyanobacteria (Soo et al., 2014) that is motile, non-photosynthetic, and capable of synthesizing several $\mathrm{B}$ and $\mathrm{K}$ vitamins (Di Rienzi et al., 2013).

\section{"Most Wanted" Species in Stool Metagenomic Data}

We decided to focus on a single bin in more detail, and thus set out to identify the genomes of most interest. Fodor et al. (2012) composed a list of the "most wanted" taxa in the human microbiota, focusing on those that were prevalent in HMP volunteers and distantly related to already sequenced organisms. In the years that have elapsed, 1000s of new genome sequences have been collected, so we updated the "most wanted" list by using $16 \mathrm{~S}$ data to investigate which bacterial taxa were most common amongst the gut samples from the MWRP (representative of the population from which our stool samples were collected) and which had matches in the GOLD database, which tracks current and past sequencing projects (Figure 1A). As expected, with the additional genomes in the public repository, the average similarity to sequenced genomes increased from $91.4 \%$ in Fodor's HMP sample to $91.9 \%$ in our MWRP sample. Based on Fodor's criteria (prevalence $\geq 20 \%$ and similarity to sequenced organisms $\leq 90 \%$ ), we identified 13 "most wanted" complete and partially complete genome bins (see Supplementary Table S1).

From the genome bins deemed essentially and partially complete, we are focusing on a single bin with a $16 \mathrm{~S}$ rRNA sequence closely matching an OTU from our updated "most wanted" taxa. The OTU was identified as being from the genus Butyricicoccus, with a prevalence of $97.5 \%$ in the MWRP and a sequence identity of $90.0 \%$ to the most related genome in the GOLD-Human and HMP databases. The genome bin was originally constructed using supervised assembly with MetaHIT metagenomic species MGS-46, and re-assembly was then performed.

This bin contained three genomes, one each from samples $\mathrm{N} 15, \mathrm{~N} 54$, and K4410, and we began by investigating their taxonomical niche. Phylogenies based on both 16S RNA, good for resolving taxa at the species level and above, and wholegenome tetranucleotide spectrum data, good for resolving taxa below the species level, confirmed that the three genomes were most closely related to the species Butyricicoccus pullicaecorum and Eubacterium desmolans (Figure 1B). The ANIb among the three genomes varied from 94.4 to $96.3 \%$, and they exhibited less than $76 \%$ similarity to the B. pullicaecorum and E. desmolans genomes (Supplementary Figure S1; Supplementary Table S3). Thus, these three genomes probably belong to the same species (the ANIb species cutoff is typically around 95\%), a species that is quite distinct from B. pullicaecorum and E. desmolans. B. pullicaecorum is the sole known member of the genus Butyricicoccus, named for its ability to produce the antiinflammatory SCFA butyrate. By contrast, E. desmolans belongs to a large and phylogenetically diverse genus that is the second most common in the human intestinal tract after Bacteroides. The Eubacterium genus as a whole is distinguished primarily by its negative metabolic characteristics (Schwiertz et al., 2000), but this particular species is known for its production of a steroid desmolase (Bokkenheuser et al., 1986). Because the assembled genomes' closest known neighbors are both clostridial cluster IV/C. leptum subgroup species, we conclude that they belong to a 
TABLE 1 | Characteristics of the three assembled Butyricicoccus sp. B MC genomes.

\begin{tabular}{lcccc}
\hline Genome & Assembled length (bp) & G + C\% & Predicted genes & Estimated completeness (\%) \\
\hline K4410 & 1877201 & 55.64 & 1,810 & 96.9 \\
N15 & 1604539 & 55.01 & 1,543 & 80.8 \\
N54 & 1452273 & 55.01 & 1,412 & 97.3 \\
\hline
\end{tabular}

${ }^{a}$ Calculated using Prodigal and HMMER version 3.0 (Eddy, 2011). We identified each bin's essential genes using the hidden Markov models (HMM) used for essentiality tests in Albertsen et al. (2013), and we declared a bin "essentially complete" if we found 100 or more unique genes from this HMM set.

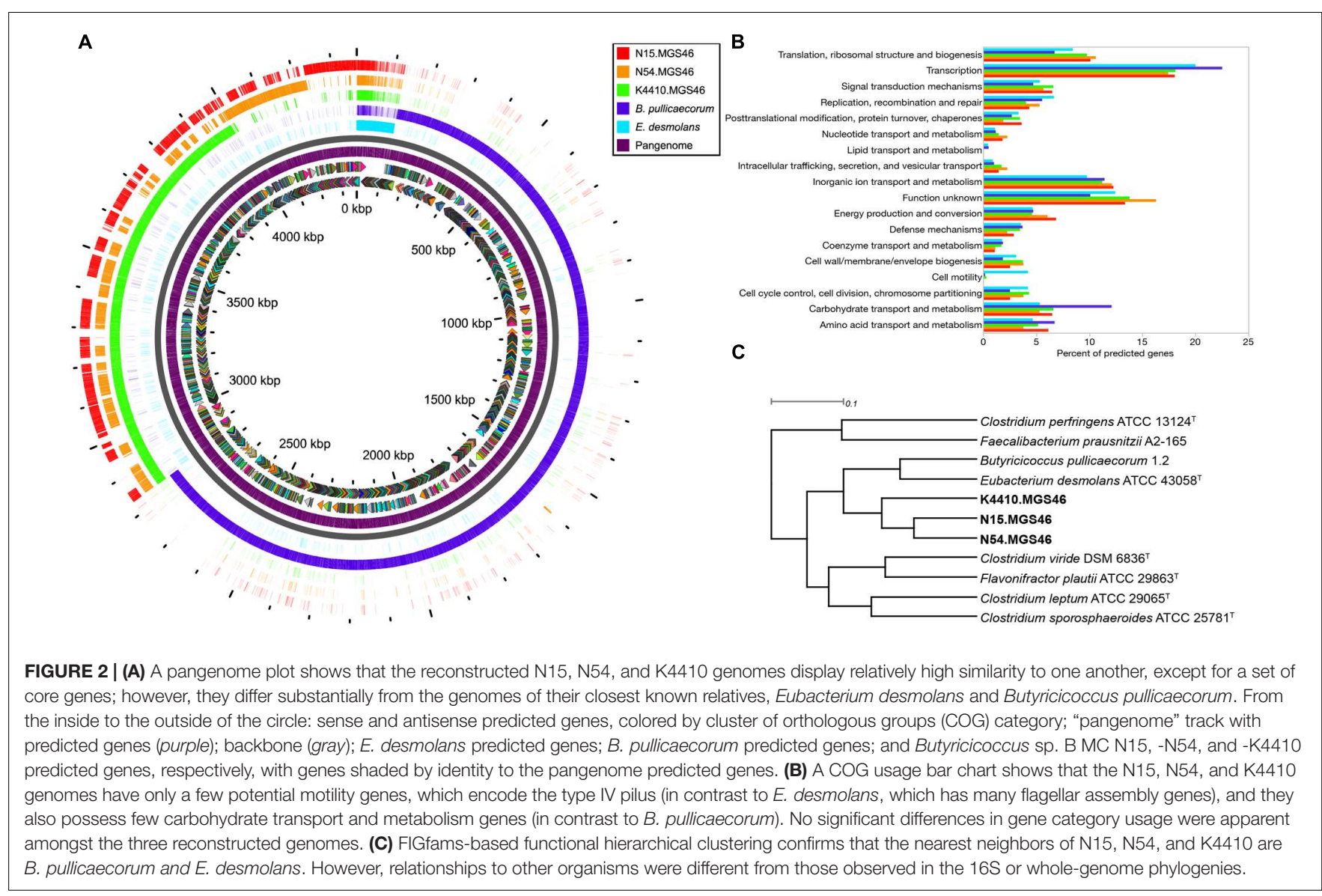

novel clostridial cluster IV species. In keeping with the naming strategy proposed by Marakeby et al. (2014), which is based on genetic similarity, we designate these strains Butyricicoccus sp. B MC-N15, -N54, and -K4410. Here, the B indicates an ANI of approximately $70 \%$ from the nearest species, the MC indicates that the Mayo Clinic generated the sequences, and the final labels indicate the sample ID. From this point on, we will refer to the strains/genomes as N15, N54, and K4410 for simplicity.

\section{The N15, N54, and K44 Genomes}

The K4410 and N54 genomes were each over 95\% complete, whereas the N15 genome was roughly $80 \%$ complete (Table 1). The genomes encoded between 1,412 and 1,810 predicted genes, and GC content was roughly $55 \%$ for each.

To learn more about the contents of the three assembled genomes, we performed a pangenome analysis in which N15,
N54, and K44 were compared to one another and to the genomes of their closest known neighbors, B. pullicaecorum and E. desmolans (Figure 2A). The genomic content of the three strains was quite similar. By contrast, there was very little genomic overlap between these strains and their nearest neighbors. The pangenome analysis matches only genes with a BLAST identity of $80 \%$ or higher; the extensive white spaces in the comparisons of N15, N54, and K44 with B. pullicaecorum and E. desmolans underscore how distantly related this novel species is from currently sequenced organisms.

Next, we analyzed gene function. A COG usage bar chart illustrates the metabolic differences between N15, N54, K44, and their nearest neighbors (Figure 2B). Most notably, genes associated with cell motility are largely absent in the assembled genomes. They do contain genes for type IV pili, which allow gliding/twitching motion (Table 2), but unlike the E. desmolans genome, they do not possess genes for flagellar assembly. In 
TABLE 2 | Genes of interest in the three assembled Butyricicoccus sp. B MC genomes ${ }^{a}$.

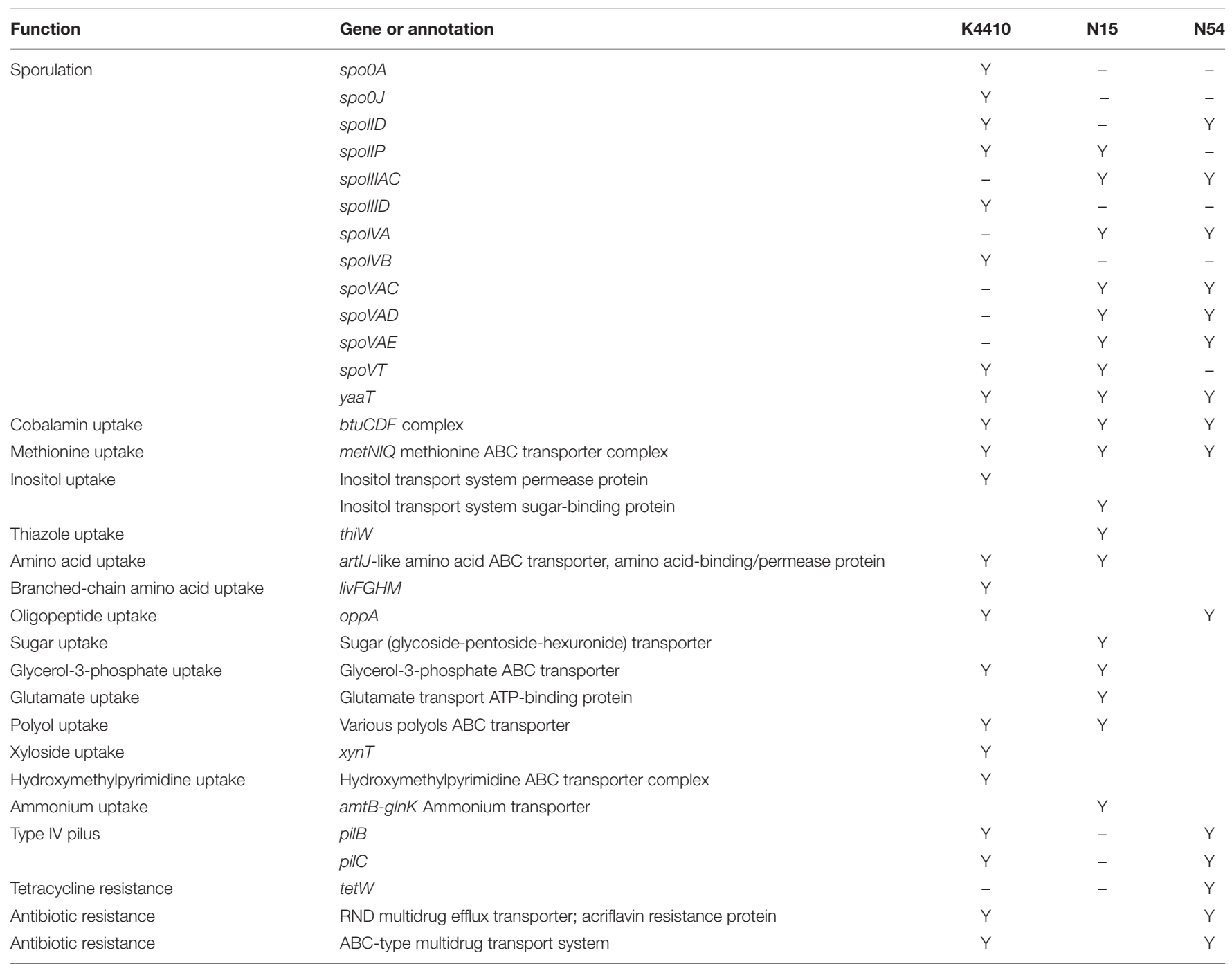

aAbbreviations: Y, yes (present); -, not present.

addition, the number of genes associated with carbohydrate transport and metabolism is distinctly lower in the three assembled genomes than in B. pullicaecorum, which degrades carbohydrates. Amongst the N15, N54, K44 genomes, no significant differences in metabolic function stood out. When the novel strains were clustered with other clostridial cluster IV/C. leptum subgroup strains based on functional gene families, their two closest neighbors remained B. pullicaecorum and E. desmolans, despite substantial differences in gene content and function (Figure 2C). However, the relationships between the three novel genomes and more distantly related organisms were different from those observed in the $16 \mathrm{~S}$ and wholegenome phylogenies; for example, Clostridium sporosphaeroides ATCC 25781 clustered quite near the three strains in the whole-genome phylogeny, but appeared not related in the functional analysis.

Table 2 lists a number of interesting genes identified in the N15, N54, and K4410 genomes. As in many of the
Clostridia (Al-Hinai et al., 2015), a predominantly complete sporulation program, including the associated sigma factors, is present. In addition, a number of genes encode transporters that allow these strains to take up various nutrients from the environment. For examples, genes for cobalamin and methionine uptake are present in all three genomes, allowing this species to avoid the metabolically costly synthesis of these molecules. In addition, genes to take up branched-chain amino acids, oligopeptides, sugars, polyols, xyloside, glycerol3-phosphate, and hydroxymethylpyrimidine are present. This diverse collection of transport genes suggests that these strains are scavengers dependent on other organisms for many essential molecules. Finally, several genes that potentially confer antibiotic resistance are present. A gene for tetracycline resistance, tet $W$, was detected in N54, and several efflux pumps were found in the reconstructed genomes as well. A scan using PATRIC failed to identify any virulence genes, however. 


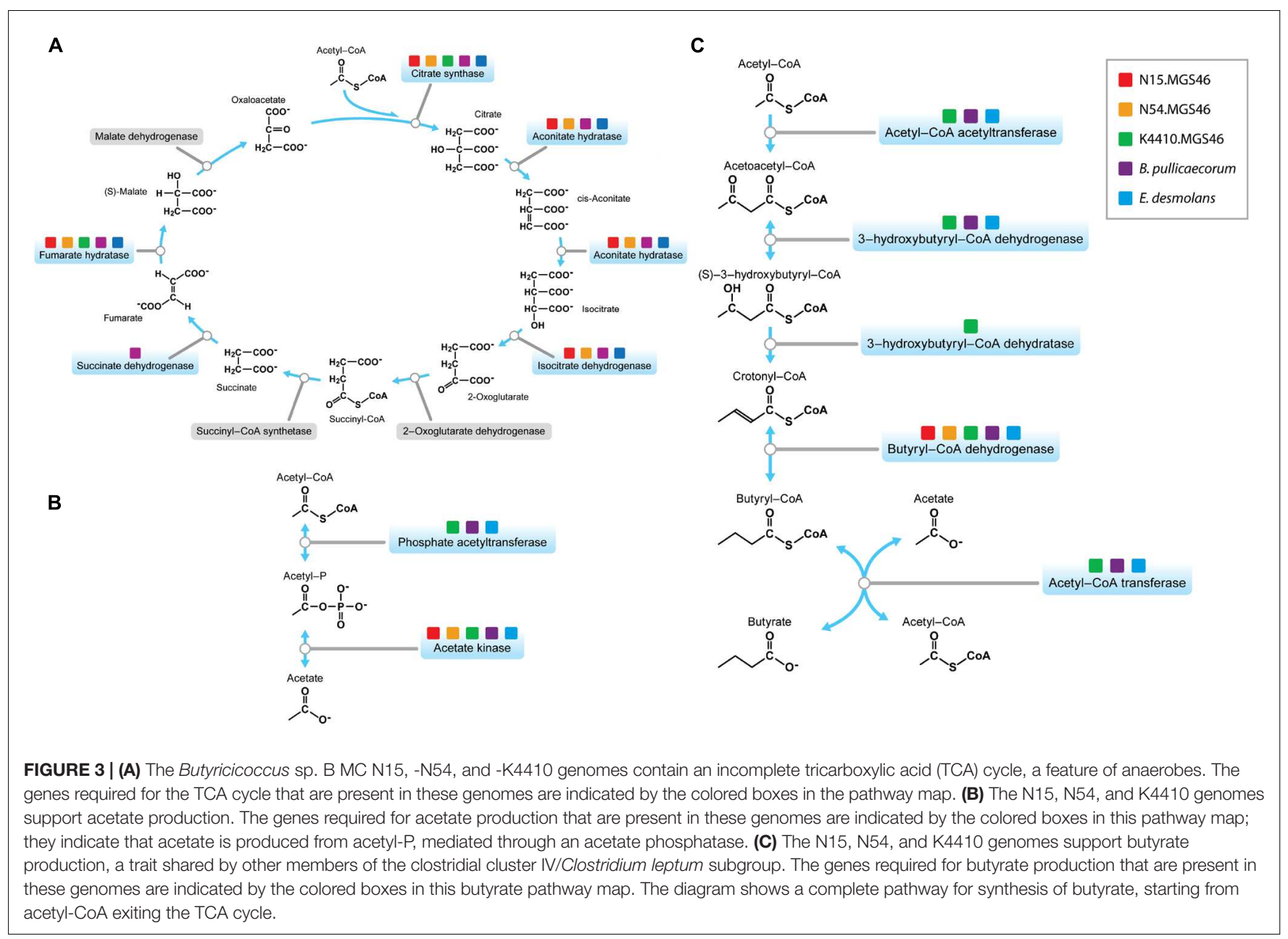

\section{Metabolism of N15, N54, and K4410}

Next, we took a more in-depth look at the metabolic genes in the three reconstructed genomes, to learn more about the potential niche of this bacterium in the human gut. We investigated whether the components of the tricarboxylic acid (TCA) cycle, a series of reactions essential for aerobic respiration, were intact. The cycle was incomplete (Figure 3A), with a pathway structure similar to that of E. desmolans and B. pullicaecorum. The genomes also lacked a gene for catalase (Supplementary Table S2), which protects aerobic organisms from oxidative damage. Thus, as expected from an intestinal member of the clostridia, these species are anaerobic.

Two of the main metabolic products of anaerobic fermentation by gut bacteria are the SCFAs acetate and butyrate, which modulate key processes in the gastrointestinal tract related to digestion and inflammation, and hence influence gastrointestinal health (Vinolo et al., 2011). Both of the strains' nearest neighbors, E. desmolans, (Morris et al., 1986) and B. pullicaecorum (Geirnaert et al., 2014), produce acetate and butyrate. Therefore, we suspected that the three reconstructed genomes would encode the ability to produce these SCFAs as well. We first examined the potential of the N15, N54, and K4410 genomes to produce acetate. In this case, the gene for acetate kinase, the protein needed to convert acetyl-P to acetate, was present (Figure 3B), suggesting that the organism excretes the compound in the gut. Next, we determined whether the genomes support the production of butyrate. The five proteins necessary to synthesize this SCFA, starting from acetyl-CoA exiting the TCA cycle (Figure 3C), are acetyl-CoA acetyltransferase, 3-hydroxy-CoA dehydrogenase, enoyl-CoA hydratase, butyrylCoA dehydrogenase, and acetate CoA-transferase; the genes for all of these proteins were present. This suggests that the organism is a butyrate producer, a trait shared by other members of the clostridial cluster IV/C. leptum subgroup.

\section{DISCUSSION}

Here we show that using readily available sequencing and analytic tools, we reconstructed the genomes of novel organisms from metagenomic data gathered from human stool samples. Current techniques allow us to zero in on organisms of interest, such as the "most wanted" taxa targeted in this paper, amidst sequences from thousands of other organisms. We exploited the following developments to reassemble essentially complete genomes from this complex sequencing environment: 
(1) We moved beyond paired-end sequencing to obtain longer, more reliable contigs, which make it easier for binning algorithms to separate organisms (Sharon et al., 2015; Kuleshov et al., 2016); and (2) We employed algorithms to quantify the completeness of candidate genomes based on conserved features (Parks et al., 2015), a strategy that works reasonably well even for novel organisms. By employing similar strategies, researchers should make rapid progress in sequencing and learning more about the HMP's "most wanted" taxa and other uncultured organisms of interest to the research community.

The three complete genomes reconstructed here belong to a previously undescribed taxon. They are more than $94 \%$ similar to one another in terms of ANI; thus, it is appropriate to group them as representatives of a single species, one that we have designated Butyricicoccus sp. B MC.

Although 16S rRNA and whole-genome phylogenies place this species between $B$. pullicaecorum and E. desmolans, in the clostridial cluster IV/C. leptum subgroup of the Clostridiales, pangenome analysis indicates that the genomes of N15, N54, and K4410 differ substantially from those of their nearest neighbors in terms of both genes and functions. With a maximum ANI of $76.1 \%$ to other sequenced genomes, these strains are novel and unique additions to clostridial cluster IV.

Functional analysis of the N15, N54, and K4410 genomes suggests that this species is an anaerobe that occupies a scavenging niche in the gastrointestinal tract and produces butyrate. First, the species does not possess the molecules needed to complete the TCA cycle, and it does not encode catalase, an enzyme that protects aerobes from the toxic byproducts of oxygen metabolism. Second, the organism probably attaches to others cells in the gut. Genes for type IV pili/fimbriae are present; these sticky filaments are used to generate twitching motions, and they play an important role in attachment to and colonization of host cells (Mattick, 2002). Reliance on other cells, whether our own or those of other bacteria, is consistent with the lack of genes related to metabolic functions such as carbohydrate transport and metabolism. Third, despite this reliance, this species does not appear to be pathogenic: we found no evidence of virulence genes, and only several antibiotic resistance genes. Fourth, the species appears to produce the anti-inflammatory SCFAs acetate and butyrate, as it possesses complete or nearly complete pathways for both. Butyrate in particular is considered a "good" fatty acid, and researchers have been experimenting with the use of butyrate-producing bacteria to treat inflammatory bowel disease (Immerseel et al., 2010).

Of course, these are all genome-based predictions, and culturing these and other poorly understood organisms is an essential step in learning more about these bacteria, their metabolic and ecological niches, and their potential uses in medicine (Stewart, 2012). However, a genome sequence is a valuable tool when setting out to culture a novel bacterium. We observed that N15, N54, and K4410 are predicted to take up vitamin B12 (cobalamin) and methionine using the same transporter system. Uptake of the amino acid methionine is very common in bacteria; cobalamin is not only used as a cofactor for methionine biosynthesis, but all three genomes surveyed here also showed evidence for a B12-dependent radical SAM protein. This finding may provide a strategy for culturing Butyricicoccus sp. B MC: Supplementation with methionine and cobalamin may facilitate this secondary degrader's growth. In addition, having a genome sequence on hand may enable researchers to synthesize probes useful for isolating the bacterium.

Our approach uses mate-pair sequencing to provide additional information for jumping large repeat gaps in the assembly process. While there are still repeats that cannot be resolved using this technique, using mate-pair libraries allows researchers to obtain scaffolds longer than previously possible. As an important limitation to note, this requires a significant amount of DNA and is limited to a subset of sequencing core providers. In addition, although mate-pair sequencing improves the quality of assembly, using longer contigs does not guarantee that contamination or misassembly will not occur. Thus, researchers will want to balance the quality of metagenomic assembly with the ability to sequence a larger number of samples, as the chance of obtaining multiple bins (albeit with shorter contigs) grows with the number of samples analyzed.

\section{CONCLUSION}

We have obtained essentially complete genomes from stool metagenomic data using readily available methods, and that these genomes provide a rich source of culture-independent data about the "dark matter" of the human microbiome. Sequence data can be mined to generate a metabolic overview of an organism, which provides insight into its niche in the human body and what factors may be needed to culture it. Thus, the strategy presented here can be used to rapidly learn more about the HMP's "most wanted" taxa, as well as poorly understood agents of disease. A concerted effort to sequence the genomes of these key players in the human microbiome is bound to lead to greater understanding of their roles in the gastrointestinal tract, and with luck, to information that can be used to maintain health and treat disease.

\section{AUTHOR CONTRIBUTIONS}

PJ and NC planned and designed the research; DA and LB collected samples; $\mathrm{AH}$ prepared the libraries and processed Illumina amplicon sequencing data; $\mathrm{PJ}, \mathrm{XC}$, and $\mathrm{HN}$ performed bioinformatic analyses; PJ, NG, BW, HN, and NC analyzed the data and wrote the paper; and all authors read and approved the final manuscript.

\section{FUNDING}

We acknowledge funding from the National Cancer Institute under grant number R01CA179243, the Center for Individualized Medicine at Mayo Clinic, and the Mayo Illinois Strategic Alliance for Technology-based Healthcare. 


\section{ACKNOWLEDGMENTS}

We thank Helena Mendes Soares and Jessica Saw for critical input on the manuscript and Kristin Harper for help preparing the manuscript.

\section{REFERENCES}

Albertsen, M., Hugenholtz, P., Skarshewski, A., Nielsen, K. L., Tyson, G. W., and Nielsen, P. H. (2013). Genome sequences of rare, uncultured bacteria obtained by differential coverage binning of multiple metagenomes. Nat. Biotechnol. 31, 533-538. doi: 10.1038/nbt.2579

Al-Hinai, M., Jones, S., and Papoutsakis, E. (2015). The Clostridium sporulation programs: diversity and preservation of endospore differentiation. Microbiol. Mol. Biol. Rev. 79, 19-37. doi: 10.1128/MMBR.00025-14

Alneberg, J., Bjarnason, B. S., de Bruijn, I., Schirmer, M., Quick, J., Ijaz, U. Z., et al. (2013). CONCOCT: Clustering cONtigs on COverage and ComposiTion. arXiv: 1312.4038

Altschul, S., Gish, W., Miller, W., Myers, E., and Lipman, D. (1990). Basic local alignment search tool. J. Mol. Biol. 215, 403-410. doi: 10.1016/S00222836(05)80360-2

Bankevich, A., Nurk, S., Antipov, D., Gurevich, A. A., Dvorkin, M., Kulikov, A. S., et al. (2012). SPAdes: a new genome assembly algorithm and its applications to single-cell sequencing. J. Comput. Biol. 19, 455-477. doi: $10.1089 / \mathrm{cmb} .2012 .0021$

Bodenhofer, U., Kothmeier, A., and Hochreiter, S. (2011). APCluster: an R package for affinity propagation clustering. Bioinformatics 27, 2463-2464. doi: 10.1093/bioinformatics/btr406

Boisvert, S., Raymond, F., Godzaridis, E., Laviolette, F., and Corbeil, J. (2012). Ray Meta: scalable de novo metagenome assembly and profiling. Genome Biol. 13:R122. doi: 10.1186/gb-2012-13-12-r122

Bokkenheuser, V., Winter, J., Morris, G., and Locascio, S. (1986). Steroid desmolase synthesis by Eubacterium desmolans and Clostridium cadavaris. Appl. Environ. Microbiol. 52, 1153-1156.

Bolger, A. M., Lohse, M., and Usadel, B. (2014). Trimmomatic: a flexible trimmer for Illumina sequence data. Bioinformatics 30, 2114-2120. doi: 10.1093/bioinformatics/btu170

Brettin, T., Davis, J. J., Disz, T., Edwards, R. A., Gerdes, S., Olsen, G. J., et al. (2015). RASTtk: a modular and extensible implementation of the RAST algorithm for building custom annotation pipelines and annotating batches of genomes. Sci. Rep. 5:8365. doi: 10.1038/srep08365

Caporaso, J., Bittinger, K., Bushman, F., DeSantis, T., Andersen, G., and Knight, R. (2010). PyNAST: a flexible tool for aligning sequences to a template alignment. Bioinformatics 26, 266-267. doi: 10.1093/bioinformatics/btp636

Chen, J., Ryu, E., Hathcock, M., Ballman, K., Chia, N., Olson, J. E., et al. (2016) Impact of demographics on human gut microbial diversity in a US Midwest population. PeerJ 4:e1514. doi: 10.7717/peerj.1514

Chu, J., Sadeghi, S., Raymond, A., Jackman, S., Nip, K., Mar, R., et al. (2014). BioBloom tools: fast, accurate and memory-efficient host species sequence screening using bloom filters. Bioinformatics 30, 3402-3404. doi: 10.1093/bioinformatics/btu558

Cole, J. R., Wang, Q., Cardenas, E., Fish, J., Chai, B., Farris, R. J., et al. (2009). The Ribosomal Database Project: improved alignments and new tools for rRNA analysis. Nucleic Acids Res. 37, D141-D145. doi: 10.1093/nar/gkn879

Crusoe, M. R., Alameldin, H. F., Awad, S., Boucher, E., Caldwell, A., Cartwright, R., et al. (2015). The khmer software package: enabling efficient nucleotide sequence analysis [version 1; referees: 2 approved, 1 approved with reservations]. F1000 Res. 4:900. doi: 10.12688/f1000research.6924.1

Dao, M., Everard, A., Aron-Wisnewsky, J., Sokolovska, N., Prifti, E., Verger, E., et al. (2016). Akkermansia muciniphila and improved metabolic health during a dietary intervention in obesity: relationship with gut microbiome richness and ecology. Gut 65, 426-436. doi: 10.1136/gutjnl-2014-308778

Di Rienzi, S. C., Sharon, I., Wrighton, K. C., Koren, O., Hug, L. A., Thomas, B. C., et al. (2013). The human gut and groundwater harbor non-photosynthetic bacteria belonging to a new candidate phylum sibling to Cyanobacteria. eLife 2:e01102. doi: 10.7554/eLife.01102

\section{SUPPLEMENTARY MATERIAL}

The Supplementary Material for this article can be found online at: http://journal.frontiersin.org/article/10.3389/fmicb. 2016.00783

Eddy, S. R. (2011). Accelerated Profile HMM Searches. PLoS Comput. Biol. 7:e1002195. doi: 10.1371/journal.pcbi.1002195

Edgar, R. C. (2010). Search and clustering orders of magnitude faster than BLAST. Bioinformatics 26, 2460-2461. doi: 10.1093/bioinformatics/btq461

Eren, A. M., Esen, Ö. C., Quince, C., Vineis, J. H., Morrison, H. G., Sogin, M. L., et al. (2015). Anvi'o: an advanced analysis and visualization platform for 'omics data. PeerJ 3:e1319. doi: 10.7717/peerj.1319

Fierer, N., Hamady, M., Lauber, C., and Knight, R. (2008). The influence of sex, handedness, and washing on the diversity of hand surface bacteria. Proc. Natl. Acad. Sci. U.S.A. 105, 17994-17999. doi: 10.1073/pnas.08079 20105

Fodor, A. A., DeSantis, T. Z., Wylie, K. M., Badger, J. H., Ye, Y., Hepburn, T., et al. (2012). The "most wanted" taxa from the human microbiome for whole genome sequencing. PLoS ONE 7:e41294. doi: 10.1371/journal.pone.0041294

Frey, B. J., and Dueck, D. (2007). Clustering by passing messages between data points. Science 315, 972-976. doi: 10.1126/science.1136800

Geirnaert, A., Steyaert, A., Eeckhaut, V., Debruyne, B., Arends, J., Van Immerseel, F., et al. (2014). Butyricicoccus pullicaecorum, a butyrate producer with probiotic potential, is intrinsically tolerant to stomach and small intestine conditions. Anaerobe 30, 70-74. doi: 10.1016/j.anaerobe.2014.08.010

Gevers, D., Knight, R., Petrosino, J. F., Huang, K., McGuire, A. L., Birren, B. W., et al. (2012). The Human Microbiome Project: a community resource for the healthy human microbiome. PLoS Biol. 10:e1001377. doi: 10.1371/journal.pbio.1001377

Handelsman, J. (2004). Metagenomics: application of genomics to uncultured microorganisms. Microbiol. Mol. Biol. Rev. 68, 669-685. doi: 10.1128/MMBR.68.4.669-685.2004

Handelsman, J., Rondon, M. R., Brady, S. F., Clardy, J., and Goodman, R. M. (1998). Molecular biological access to the chemistry of unknown soil microbes: a new frontier for natural products. Chem. Biol. 5, R245-R249. doi: 10.1016/S10745521(98)90108-9

Huerta-Cepas, J., Szklarczyk, D., Forslund, K., Cook, H., Heller, D., Walter, M. C., et al. (2015). eggNOG 4.5: a hierarchical orthology framework with improved functional annotations for eukaryotic, prokaryotic and viral sequences. Nucleic Acids Res. 44, D286-D293. doi: 10.1093/nar/gkv1248

Hyatt, D., LoCascio, P. F., Hauser, L. J., and Uberbacher, E. C. (2012). Gene and translation initiation site prediction in metagenomic sequences. Bioinformatics 28, 2223-2230. doi: 10.1093/bioinformatics/bts429

Immerseel, F. V., Ducatelle, R., Boon, N., Wiele, T. V. D., Verbeke, K., Rutgeerts, P., et al. (2010). Butyric acid-producing anaerobic bacteria as a novel probiotic treatment approach for inflammatory bowel disease. J. Med. Microbiol. 59(Pt 2), 141-143. doi: 10.1099/jmm.0.017541-0

Jeraldo, P., Hernández, A., White, B., O’Brien, D., Ahlquist, D., Boardman, L., et al. (2015). Draft genome sequences of 24 microbial strains assembled from direct sequencing from 4 stool samples. Genome Announc. 3:e5265. doi: 10.1128/genomeA.00526-15

Kuleshov, V., Jiang, C., Zhou, W., Jahanbani, F., Batzoglou, S., and Snyder, M. (2016). Synthetic long-read sequencing reveals intraspecies diversity in the human microbiome. Nat. Biotechnol. 34, 64-69. doi: 10.1038/nbt.3416

Lagesen, K., Hallin, P., Rødland, E. A., Staerfeldt, H.-H., Rognes, T., and Ussery, D. W. (2007). RNAmmer: consistent and rapid annotation of ribosomal RNA genes. Nucleic Acids Res. 35, 3100-3108. doi: 10.1093/nar/gkm160

Li, H., and Durbin, R. (2009). Fast and accurate short read alignment with Burrows-Wheeler transform. Bioinformatics 25, 1754-1760. doi: 10.1093/bioinformatics/btp324

Li, H., Handsaker, B., Wysoker, A., Fennell, T., Ruan, J., Homer, N., et al. (2009). The Sequence Alignment/Map format and SAMtools. Bioinformatics 25, 20782079. doi: 10.1093/bioinformatics/btp352

Lok, C. (2015). Mining the microbial dark matter. Nature 522, 270-273. doi: $10.1038 / 522270$ a 
Marakeby, H., Badr, E., Torkey, H., Song, Y., Leman, S., Monteil, C. L., et al. (2014). A system to automatically classify and name any individual genome-sequenced organism independently of current biological classification and nomenclature. PLoS ONE 9:e8912. doi: 10.1371/journal.pone.0089142

Martínez, I., Lattimer, J., Hubach, K., Case, J., Yang, J., Weber, C., et al. (2013). Gut microbiome composition is linked to whole grain-induced immunological improvements. ISME J. 7, 268-280. doi: 10.1038/ismej.2012.104

Mattick, J. (2002). Type IV pili and twitching motility. Annu. Rev. Microbiol. 56, 289-314. doi: 10.1146/annurev.micro.56.012302. 160938

Meyer, F., Overbeek, R., and Rodriguez, A. (2009). FIGfams: yet another set of protein families. Nucleic Acids Res. 37, 6643-6654. doi: 10.1093/nar/ gkp698

Morris, G. N., Winter, J., Cato, E. P., Ritchie, A. E., and Bokkenheuser, V. D. (1986). Eubacterium desmolans sp. nov., a steroid desmolase-producing species from cat fecal flora. Int. J. Syst. Bacteriol. 36, 183-186. doi: 10.1099/00207713-36-2183

Nielsen, H. B., Almeida, M., Juncker, A. S., Rasmussen, S., Li, J., Sunagawa, S., et al. (2014). Identification and assembly of genomes and genetic elements in complex metagenomic samples without using reference genomes. Nat. Biotechnol. 32, 822-828. doi: 10.1038/nbt.2939

Parks, D. H., Imelfort, M., Skennerton, C. T., Hugenholtz, P., and Tyson, G. W. (2015). CheckM: assessing the quality of microbial genomes recovered from isolates, single cells, and metagenomes. Genome Res. 25, 1043-1055. doi: 10.1101/gr.186072.114

Petkau, A., Stuart-Edwards, M., Stothard, P., and Van Domselaar, G. (2010). Interactive microbial genome visualization with GView. Bioinformatics 26, 3125-3126. doi: 10.1093/bioinformatics/btq588

Pignatelli M. (2014). Blast2lca. Available at: https://github.com/emepyc/Blast2lca

Price, M. N., Dehal, P. S., and Arkin, A. P. (2010). FastTree 2 - approximately maximum-likelihood trees for large alignments. PLoS ONE 5:e9490. doi: 10.1371/journal.pone.0009490

Qin, J., Li, R., Raes, J., Arumugam, M., Burgdorf, K. S., Manichanh, C., et al. (2010). A human gut microbial gene catalogue established by metagenomic sequencing. Nature 464, 59-65. doi: 10.1038/nature08821

Quast, C., Pruesse, E., Yilmaz, P., Gerken, J., Schweer, T., Yarza, P., et al. (2013). The SILVA ribosomal RNA gene database project: improved data processing and web-based tools. Nucleic Acids Res. 41, D590-D596. doi: 10.1093/nar/gks1219

Quinlan, A. R. (2014). BEDTools: The Swiss-Army tool for genome feature analysis. Curr. Protoc. Bioinformatics 47, 11.12.11-11.12.34. doi: 10.1002/0471250953.bi1112s47

Reddy, T. B. K., Thomas, A. D., Stamatis, D., Bertsch, J., Isbandi, M., Jansson, J., et al. (2015). The Genomes OnLine Database (GOLD) v.5: a metadata management system based on a four level (meta)genome project classification. Nucleic Acids Res. 43, D1099-D1106. doi: 10.1093/nar/gku950

Richter, M., and Rosselló-Móra, R. (2009). Shifting the genomic gold standard for the prokaryotic species definition. Proc. Natl. Acad. Sci. U.S.A. 106, 19126 19131. doi: 10.1073/pnas.0906412106

Rosselló-Móra, R., and Amann, R. (2015). Past and future species definitions for Bacteria and Archaea. Syst. Appl. Microbiol. 38, 209-216. doi: 10.1016/j.syapm.2015.02.001

Ruby, J. G., Bellare, P., and Derisi, J. L. (2013). PRICE: software for the targeted assembly of components of (Meta) genomic sequence data. G 3, 865-880. doi: $10.1534 / g 3.113 .005967$
Schwiertz, A., Blay, G. L., and Blaut, M. (2000). Quantification of different Eubacterium spp. in human fecal samples with species-specific $16 \mathrm{~S}$ rRNAtargeted oligonucleotide probes. Appl. Environ. Microbiol. 66, 375-382. doi: 10.1128/AEM.66.1.375-382.2000

Sharon, I., and Banfield, J. F. (2013). Genomes from metagenomics. Science 342, 1057-1058. doi: 10.1126/science. 1247023

Sharon, I., Kertesz, M., Hug, L. A., Pushkarev, D., Blauwkamp, T. A., Castelle, C. J., et al. (2015). Accurate, multi-kb reads resolve complex populations and detect rare microorganisms. Genome Res. 25:gr.183012.183114. doi: 10.1101/gr.183012.114

Simpson, J. T., and Durbin, R. (2012). Efficient de novo assembly of large genomes using compressed data structures. Genome Res. 22, 549-556. doi: 10.1101/gr.126953.111

Snyder, E. E., Kampanya, N., Lu, J., Nordberg, E. K., Karur, H. R., Shukla, M., et al. (2007). PATRIC: the VBI PathoSystems Resource Integration Center. Nucleic Acids Res. 35, D401-D406. doi: 10.1093/nar/gkl858

Soo, R. M., Skennerton, C. T., Sekiguchi, Y., Imelfort, M., Paech, S. J., Dennis, P. G., et al. (2014). An expanded genomic representation of the phylum cyanobacteria. Genome Biol. Evol. 6, 1031-1045. doi: 10.1093/gbe/evu073

Stewart, E. (2012). Growing unculturable bacteria. J. Bacteriol. 194, 4151-4160. doi: 10.1128/JB.00345-12

Teeling, H., Meyerdierks, A., Bauer, M., Amann, R., and Glöckner, F. O. (2004). Application of tetranucleotide frequencies for the assignment of genomic fragments. Environ. Microbiol. 6, 938-947. doi: 10.1111/j.14622920.2004.00624.x

Tennessen, K., Andersen, E., Clingenpeel, S., Rinke, C., Lundberg, D. S., Han, J., et al. (2015). ProDeGe: a computational protocol for fully automated decontamination of genomes. ISME J. 10, 269-272. doi: 10.1038/ismej.2015.100

Torsvik, V., Goksøyr, J., and Daae, F. (1990). High diversity in DNA of soil bacteria. Appl. Environ. Microbiol. 56, 782-787.

Vinolo, M., Rodrigues, H., Nachbar, R., and Curi, R. (2011). Regulation of inflammation by short chain fatty acids. Nutrients 3, 858-876. doi: 10.3390/nu3100858

Wood, D. E., and Salzberg, S. L. (2014). Kraken: ultrafast metagenomic sequence classification using exact alignments. Genome Biol. 15:R46. doi: 10.1186/gb2014-15-3-r46

Zackular, J., Baxter, N., Iverson, K., Sadler, W., Petrosino, J., Chen, G., et al. (2013). The gut microbiome modulates colon tumorigenesis. MBio 4:e692. doi: 10.1128/mBio.00692-13

Zerbino, D. R., and Birney, E. (2008). Velvet: algorithms for de novo short read assembly using de Bruijn graphs. Genome Res. 18, 821-829. doi: 10.1101/gr.074492.107

Conflict of Interest Statement: The authors declare that the research was conducted in the absence of any commercial or financial relationships that could be construed as a potential conflict of interest.

Copyright (C) 2016 Jeraldo, Hernandez, Nielsen, Chen, White, Goldenfeld, Nelson, Alhquist, Boardman and Chia. This is an open-access article distributed under the terms of the Creative Commons Attribution License (CC BY). The use, distribution or reproduction in other forums is permitted, provided the original author(s) or licensor are credited and that the original publication in this journal is cited, in accordance with accepted academic practice. No use, distribution or reproduction is permitted which does not comply with these terms. 